PREPARED FOR THE U.S. DEPARTMENT OF ENERGY, UNDER CONTRACT DE-AC02-76CH03073

PPPL-3917

PPPL-3917

UC-70

Collisional Transport in a Low Aspect Ratio Tokamak -Beyond the Drift Kinetic Formalism

by

D.A. Gates and R.B. White

January 2004
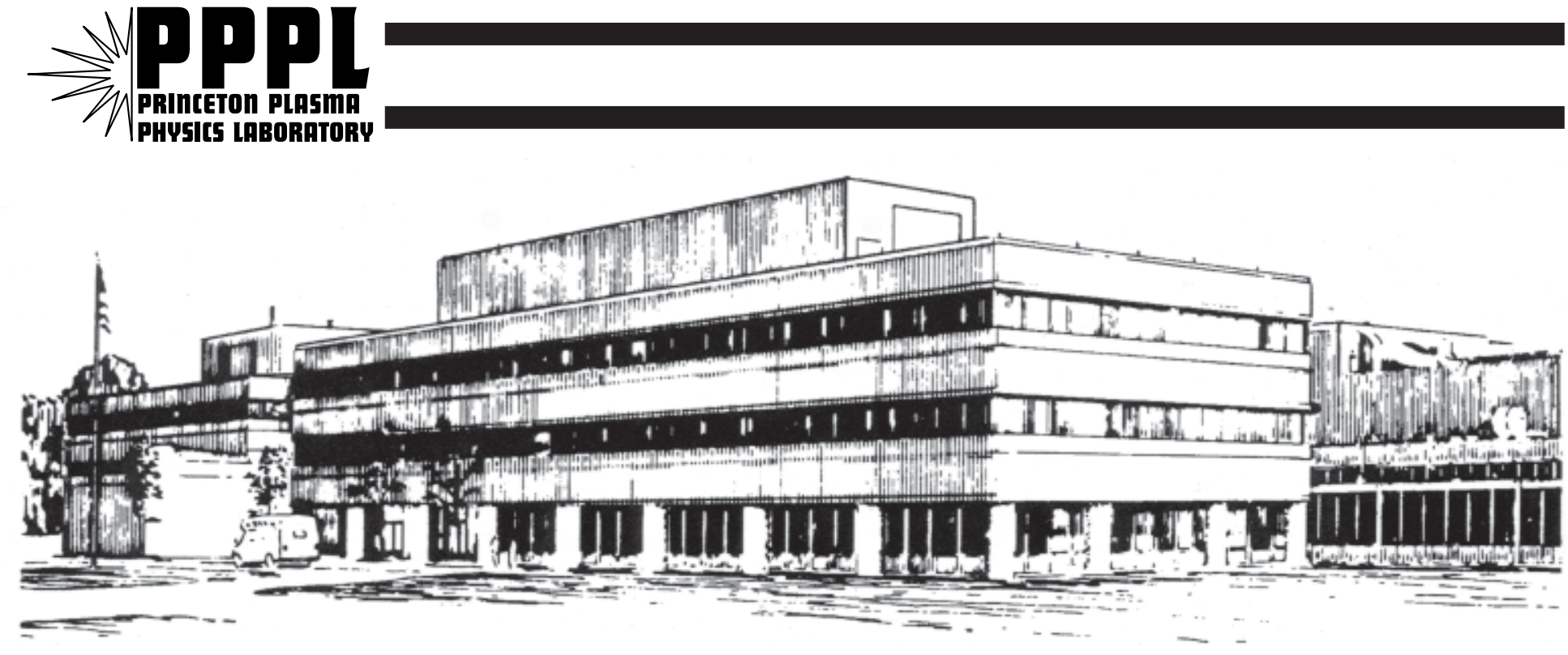

PRINCETON PLASMA PHYSICS LABORATORY PRINCETON UNIVERSITY, PRINCETON, NEW JERSEY 


\section{PPPL Reports Disclaimer}

This report was prepared as an account of work sponsored by an agency of the United States Government. Neither the United States Government nor any agency thereof, nor any of their employees, makes any warranty, express or implied, or assumes any legal liability or responsibility for the accuracy, completeness, or usefulness of any information, apparatus, product, or process disclosed, or represents that its use would not infringe privately owned rights. Reference herein to any specific commercial product, process, or service by trade name, trademark, manufacturer, or otherwise, does not necessarily constitute or imply its endorsement, recommendation, or favoring by the United States Government or any agency thereof. The views and opinions of authors expressed herein do not necessarily state or reflect those of the United States Government or any agency thereof.

\section{Availability}

This report is posted on the U.S. Department of Energy's Princeton Plasma Physics Laboratory Publications and Reports web site in Fiscal Year 2004. The home page for PPPL Reports and Publications is: http://www.pppl.gov/pub_report/

DOE and DOE Contractors can obtain copies of this report from:

U.S. Department of Energy

Office of Scientific and Technical Information

DOE Technical Information Services (DTIS)

P.O. Box 62

Oak Ridge, TN 37831

Telephone: (865) 576-8401

Fax: (865) 576-5728

Email: reports@adonis.osti.gov

This report is available to the general public from:

National Technical Information Service

U.S. Department of Commerce

5285 Port Royal Road

Springfield, VA 22161

Telephone: $1-800-553-6847$ or

(703) $605-6000$

Fax: (703) 321-8547

Internet: http://www.ntis.gov/ordering.htm 


\title{
Collisional Transport in a low aspect ratio tokamak - beyond the drift kinetic formalism*
}

\author{
D. A. Gates, R. B. White \\ Plasma Physics Laboratory, Princeton University, P.O.Box 451, \\ Princeton, New Jersey 08543
}

\begin{abstract}
Calculations of collisional thermal and particle diffusivities in toroidal magnetic plasma confinement devices order the toroidal gyroradius to be small relative to the poloidal gyro-radius, i.e $\rho_{i_{\phi}}<<\rho_{i_{\theta}}$ where $\rho_{i_{\phi}} \equiv m_{i} v_{t h_{i}} / q B_{\phi}$ and $\rho_{i_{\theta}} \equiv m_{i} v_{t h_{i}} / q B_{\theta}$. This ordering is central to what is usually referred to as neoclassical transport theory. This ordering is incorrect at low aspect ratio (with aspect ratio $A \equiv R / a$, where $R$ is the major radius of the torus and $a$ is the minor radius), where it can often be the case that $\rho_{i_{\phi}}>\rho_{i_{\theta}}$. We calculate the correction to the particle and thermal diffusivities at low aspect ratio by comparing the diffusivities as determined by a full orbit code (which we refer to as omniclassical diffusion) with those from a gyro-averaged orbit code (neoclassical diffusion). In typical low aspect ratio devices the omniclassical diffusion can be up to 2.5 times the calculated neoclassical value. We discuss the implications of this work on the anal-
\end{abstract}


ysis of collisional transport in low aspect ratio magnetic confinement experiments. 
The theoretical basis of collisional transport in high aspect ratio magnetic plasma confinement devices has been well established for at least thirty years (see, e.g. [1] ). Transport theory at high aspect ratio is based on the "small gyro-radius expansion", which assumes that $\rho_{i} / a \equiv \rho *<<1$. In addition, it is generally assumed that $\left|B_{\theta} / B_{\phi}\right|<<1$ (where $B_{\theta}$ is the poloidal magnetic field and $B_{\phi}$ is the toroidal magnetic field), which is in turn the result of two additional assumptions - $\epsilon \equiv a / R<1$ (where $a$ is the minor radius of the torus and $R$ is the major radius) and $q \equiv d \Phi / d \psi=O(1)$ (where $\Phi$ is the toroidal flux and $\psi$ is the poloidal flux). In low aspect ratio tokamaks [2], often referred to as spherical torii, the $\epsilon<<1$ assumption is explicitly violated. As a result, the assumption that $\left|B_{\theta} / B_{\phi}\right|<<1$ is also invalid. These assumptions are fundamental to the derivation of the gyro-averaged kinetic equation, also known as the drift kinetic equation. The drift kinetic equation is the basis of neoclassical transport theory.

In order to determine the magnitude of the correction to collisional transport due to finite gyro-radius, we compare the result of the gyro-average orbit code ORBIT $[3,4]$ with those from the full orbit code GYROXY [5]. We coin the word omniclassical to refer to transport calculations based on full particle orbits in genreal toroidal geometry with arbitrary aspect ratio. We explicitly 
note that omniclassical diffusion is distinct from both neoclassical diffusion, which is done in full toroidal geometry but averages over gyroradius, and classical diffusion, which includes gyro-radius but assumes straight field line geometry. The collision frequency and temperatures are set to be the same in both codes and the diffusivities are calculated. Care was taken to verify the same pitch angle scattering rate was obtained in each code, due to the very different scattering operators used. It is useful to note that the actual value of the collision frequency falls out of this ratio as long as the collisionality is well into the banana regime (i.e. $\nu_{i e}<<\omega_{b}$ ), since we are only interested in the ratio of omni- to neoclassical diffusion (and diffusion is linear in collision frequency). The correction is therefore essentially a geometric correction factor, arising from the now widely differing full and gyro-averaged orbits. We restrict ourselves to considering only modifications to the ion transport. In a real plasma, however, the collisionality regime mary vary across the minor radius, which may modify the resultant correction factor profile.

Two equilibria from the National Spherical Torus Experiment (NSTX) [6] were chosen as representative low aspect ratio equilibria. Isoflux contour plots of the equilibria used are shown in Figures 1 and 2. These particular equilibria were chosen because they have very different values of $\left|B_{\theta} / B_{\phi}\right|$, 
so as to determine in a rough manner how strongly the transport correction scales with this ratio. The double null discharge (equilbrium 1) has $B_{\phi}(0)=$ $0.3 T$ (where $B_{\phi}(0) \equiv$ the applied vacuum toroidal field at the vessel midpoint, i.e. $R=0.86 m$ ) and with $I_{p}=1.2 M A$ (where $I_{p} \equiv$ the toroidal plasma current), whereas the single null discharge (equilibrium 2) has $B_{\phi}(0)=0.45 T$ and $I_{p}=0.8 M A$. The ratio of $\left|B_{\theta} / B_{\phi}\right| \sim \mu_{0} I_{p} /\left(2 \pi a \sqrt{1+\kappa^{2}} B_{\phi}\right)$ (with $\kappa=$ $b / a$ and $b$ the plasma height) varies more than a factor of 2 between the chosen equilibria (the relevant geometric factors vary between these discharges on the order of $10 \%)$.

Representative particle orbits are shown for equilibrium 1 in Figures 3 and 4. The plot region in Figure 4 is indicated by the box in Figure 3. In Figure 3 the black trace is the full orbit, whereas the red trace is the gyroaveraged orbit. It is evident from this picture alone that the diffusion step size will vary substantially between the two models. In Figure 4, which has a plot region corresponding to the box drawn in Figure 3, the black trace is the downward drifting part of the full orbit and green is the upward drifting part, whereas the red dashed line is the gyro averaged orbit. Note that the maximum orbit width for the full orbit varies by nearly a factor of 5 over the banana width (denoted in Figure 4 as $\Delta_{b}$ for the banana width and $\Delta_{t o t}$ for 
the full orbit width).

To carry out the numerical simulations, two thousand particles with energy $1.1 \mathrm{keV}$ are deposited as a $\delta$-function in $\psi$ with uniform poloidal distribution, but with a random initial velocity space pitch angle distribution. This distribution is then allowed to evolve under the influence of pitch angle scattering. The mean square normalized flux deviation from the initial flux surface is plotted vs. time for equilibrium 1 for the flux surface with an outboard major radius located at $R=130 \mathrm{~cm}$ in Figure 5 for both the neoclassical and the full omniclassical case. After filling in the orbit widths from the initial $\delta$-function distribution (typically in one to two bounce times) the particles then diffuse radially. A line is fitted to each time sequence, the slope of which is the diffusivity and the offset is the mean orbit width in flux coordinates.

The calculation is then repeated at ten flux surfaces which are equally spaced in outboard major radius, one every $5 \mathrm{~cm}$ starting at $R=110 \mathrm{~cm}$ (thereby avoiding both the magnetic axis and the last closed flux surface). Checks of the variation of the diffusivity with collisionality in the banana regime indicate that the dependence is linear with unity slope to better than $5 \%$. Checks were also performed with analytic high aspect ratio equilibria 
to verify the omni- to neoclassical diffusivity ratio approaches one at high aspect ratio. Also, we verified that the ratio of omni- to neoclassical thermal diffusivity is the same as the equivalent ratio for the particle diffusivities to a high degree of accuracy (thermal diffusivity is calculated using a Maxwellian initial energy distribution and weighting the square of the displacement with energy).

The resultant omni- to neoclassical diffusivity ratio is plotted versus normalized poloidal flux for both equilibria 1 and 2 in Figure 6 . The peak ratio is $\sim 2.5$, for the higher current low toroidal field case (equilibrium 1 ). The higher field lower current equilibrium (equilibrium 2) also has a substantial correction over the neoclassical value with a mean value of $\sim 1.5$. The omni to neocalssical diffusivity ratio rises strongly with radius for equilibrium 1 , while for equilibrium 2 the peak correction factor is near the mid-radius. Equilibrium 1 is a paramagnetic plasma, so the toroidal field falls off faster than $1 / R$, whereas Equilibrium 2 is nearly diamagnetic giving rise to a smaller variation in toroidal field as $R$ increases. It is therefore the difference in $\beta_{p}$ (which is the source of the diamagnetism) that causes the difference in radial dependence for the diffusivity ratios.

The effect of finite gyro-radius at low aspect ratio has been investigated 
previously [7] for slowing down of fast (neutral beam) particles. In this work, the effects of finite gyro-radius on diffusion were ignored since the slowing down rate is typically larger than the pitch angle scattering rate for fast ions. The result of this work was that the gyro-averaged orbit model was sufficient for modeling fast particle slowing down (i.e. for calculating plasma heating from fast particles). The reason that diffusion is not well represented by gyro-average orbits, while slowing down is, can be simply understood. On average the region over which a particle will deposit it's energy is centered about the mean location of the particle. Since the gyro-orbit is still roughly circular, the average particle location is still very near the location of the gyro-averaged orbit (accurate to within the approximation that the field does not vary over the gyro-radius, i. e. the $\rho_{i_{\phi}} / a<<1$ ). Diffusivity, on the other hand, scales as $\chi \sim \nu_{\text {eff }} \rho_{\text {eff }}^{2}$ where $\rho_{\text {eff }}$ is a measure of the RMS deviation of a particle from a flux surface and $\nu_{e f f}$ is the effective pitch angle scattering rate. However, as is apparent from Figure 4, the RMS deviation from a flux surface varies significantly between the full and gyro-averaged orbits.

The implications of this calculation for low aspect ratio devices are wide ranging. Firstly, traditional tokamak analysis tools cannot be used to reliably predict the expected minimum level of collisional transport. Secondly, the 
modification to the transport is not a simple correction to neoclassical theory, since the finite gyro-radius assumption is fundamental to the derivation of neoclassical transport theory (although we do not rule out that a tractable correction could be constructed, thereby salvaging the substantial intellectual investment in tokamak transport analysis (as in, e.g., [8]) for use in low aspect ratio plasmas). Thirdly, and perhaps most interestingly, the omniclassical ion transport is measurably higher than predicted by neoclassical theory. This last point is particularly important given recent experimental results from NSTX that have shown ion temperatures well in excess of those predicted by neoclassical theory [9]. The omniclassical corrections calculated above motivates the re-examination of the theoretically predicted ion temperature for the plasmas given in [9], which should drop as a result of the calculated increased ion thermal diffusivity (equilibrium 2 is in fact the same equilibrium for which the neoclassical ion temperature was calculated in [9]). This will increase the magnitude of the apparent ion temperature anomaly by widening the gap between the predictions of the maximum possible ion temperature and those measured in the experiment. This increase in omniclassical thermal diffusivity therefore strengthens the argument for the possible existence of a additional ion heat source in low aspect ratio 
tokamaks heated by neutral beams (see, e.g. [10]). Similarly, comparisons to neoclassical theory in other spherical torus devices such as the Small Tight Aspect Ratio Tokamak (START) bear re-examination [11]. Finally, we note that the increased omniclassical diffusivity is very likely to lead to an increase in the calculated bootstrap current when a similar procedure is applied to that phenomena, due to the direct relation of the bootstrap current to radial transport.

The authors would like to acknowledge helpful discussions with D. R. Mikkelsen.

*This work was supported by the U.S. Department of Energy Grant under contract number DE-AC02-76CH03073. 


\section{References}

[1] F. L. Hinton and R. D. Hazeltine, Rev. Mod. Phys., 48239 (1976)

[2] Y-K. M. Peng and D. J. Strickler, Nucl. Fusion 26, 769 (1986)

[3] R. B. White and M. S. Chance Phys. Fluids 27, 2455 (1984)

[4] R. B. White, Phys. Fluids B 2, 845 (1990)

[5] R. B. White, L. Chen, Z. Lin, Phys. Plasmas 91890 (2002)

[6] M. Ono, S. M. Kaye, Y. -K. M. Peng, et al., Nucl. Fusion 40, 557 (2000)

[7] D. R. Mikkelsen, R. B. White, R. J. Akers, S. M. Kaye, D. C. McCune, J. E. Menard, Phys. Plasmas, 43667 (1997)

[8] W. A. Houlberg, K. C. Shaing, S. P. Hirshman, M. C. Zarnstorff, Phys. Plasmas, 4 $3230(1997)$

[9] D. A. Gates and the NSTX national research team, Phys. Plasmas, 101659 (2003)

[10] D. A. Gates, N. N. Gorelenkov, R. B. White, Phys. Rev. Lett. 87, 205003-1 (2001)

[11] D. A. Gates, R. Akers, L. Appel, et al., Phys. Plasmas 5, 1775 (1998) 


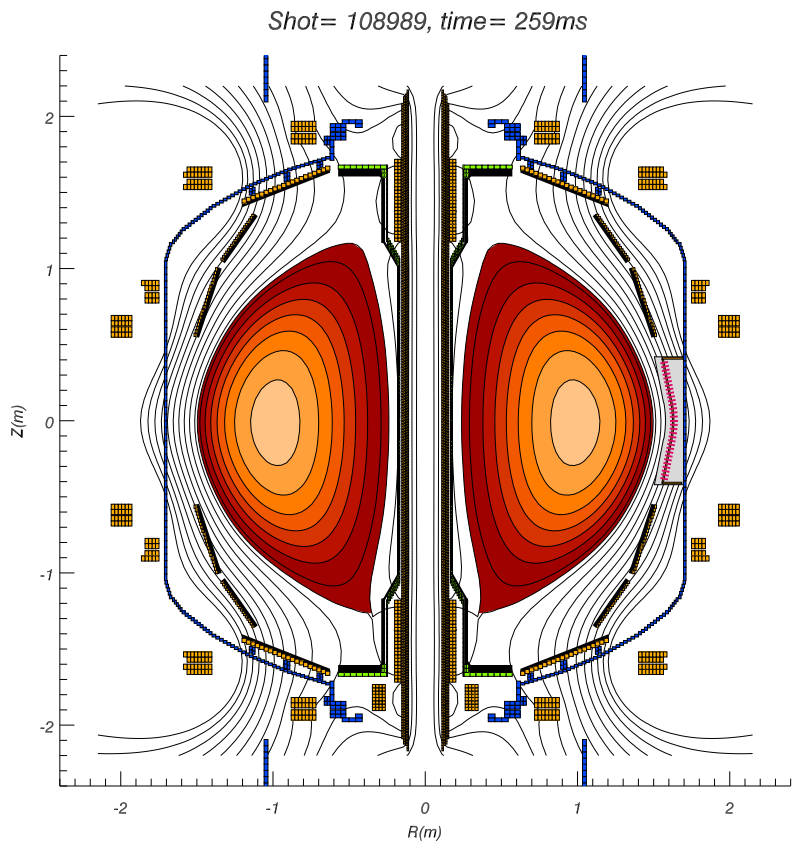

(10) $N S T X=$

Figure 1: Isoflux contour plot for NSTX shot number 108989, $B_{\phi}(R=$ $0.86 m)=0.3 T, I_{p}=1.2 M A, \beta_{t}=35 \%$ 


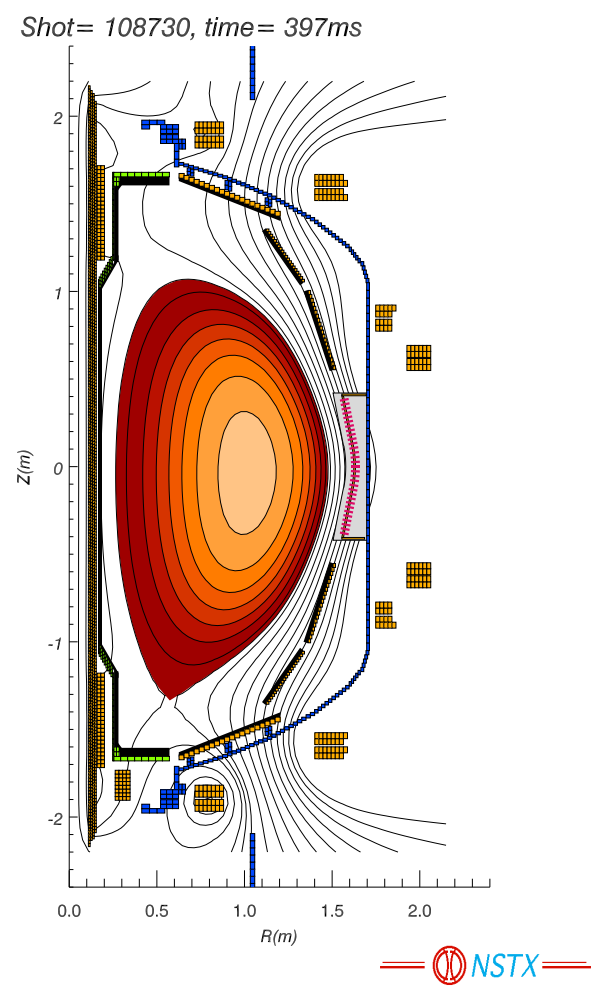

Figure 2: Isoflux contour plot for NSTX shot number $108730, B_{\phi}(R=$ $0.86 m)=0.45 T, I_{p}=0.8 M A, \beta_{t}=15 \%$ 


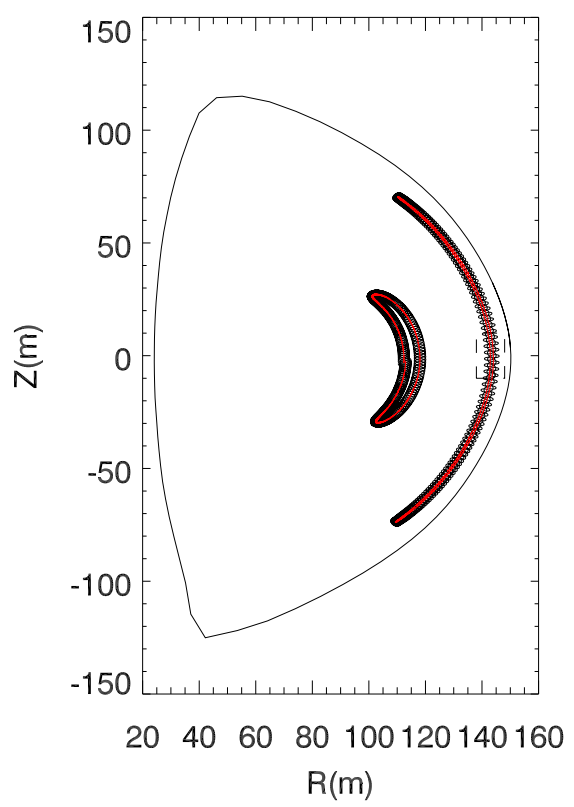

Figure 3: Representative calculated particle orbits for the full orbit code (black) and the gyro-averaged orbit code (red) for the equilibrium in Figure 1. The particle has $E=2.0 \mathrm{keV}, \lambda_{0}=v_{\|_{0}} / v=0.3$. The inner particle is born on the flux surface located at $\mathrm{R}=115 \mathrm{~cm}, \mathrm{Z}=0 \mathrm{~cm}$, while the outer orbit is born at $\mathrm{R}=145 \mathrm{~cm}, \mathrm{Z}=0 \mathrm{~cm}$ 


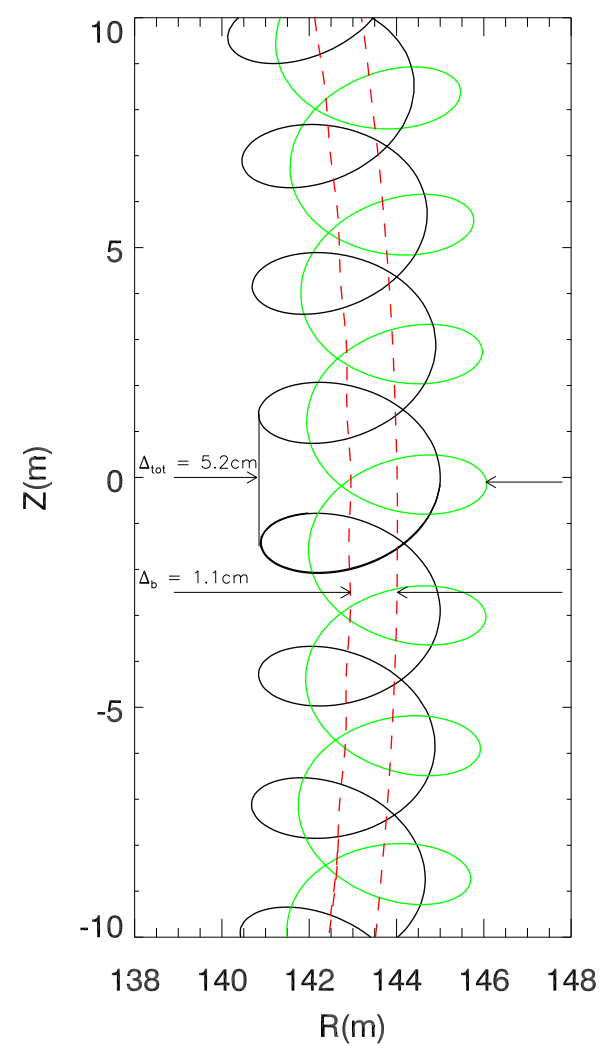

Figure 4: Expanded view of the orbits in Figure 3 (the plot region corresponds to the region of the box shown in Figure 3). Notice the large difference between the maximum orbit widths for the full orbit, $\Delta_{\text {tot }}$, and the gyroaveraged orbit $\Delta_{b}$. 


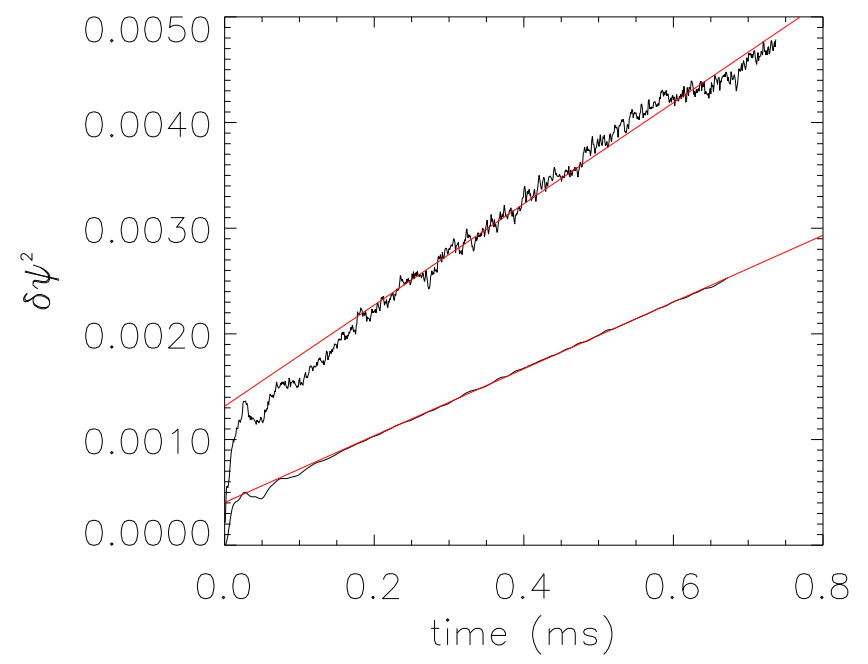

Figure 5: Time history of the mean square deviation from the starting flux surface measured in units of normalized flux. The red line is a least squares fir to the data after $\mathrm{t}=0.15 \mathrm{~ms}$. The slope of this line is the particle diffusivity in flux space. 


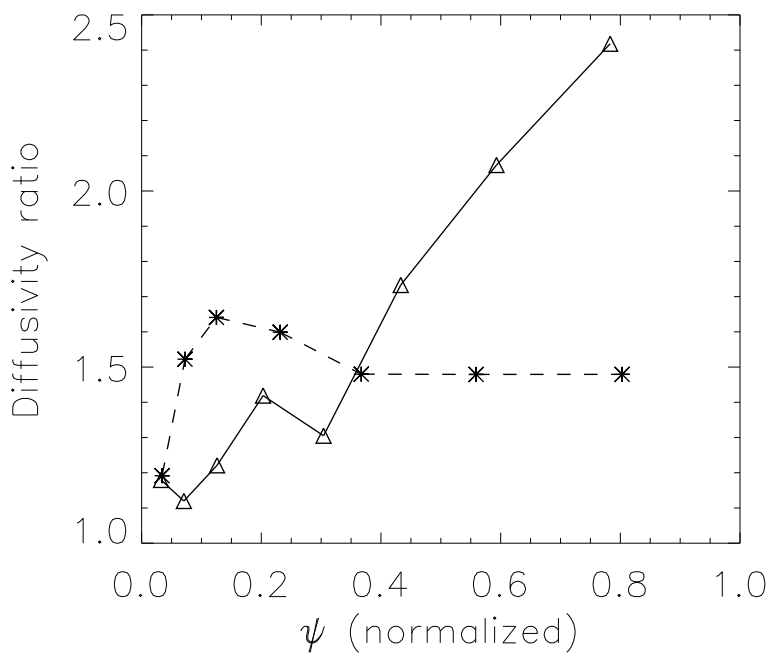

Figure 6: Radial profiles of the diffusivity correction factor due to finite gyroradius for the 2 equilibria above.The solid line is for Equilibrium 1 and the dashed is Equilibrium 2 


\section{External Distribution}

Plasma Research Laboratory, Australian National University, Australia

Professor I.R. Jones, Flinders University, Australia

Professor João Canalle, Instituto de Fisica DEQ/IF - UERJ, Brazil

Mr. Gerson O. Ludwig, Instituto Nacional de Pesquisas, Brazil

Dr. P.H. Sakanaka, Instituto Fisica, Brazil

The Librarian, Culham Laboratory, England

Mrs. S.A. Hutchinson, JET Library, England

Professor M.N. Bussac, Ecole Polytechnique, France

Librarian, Max-Planck-Institut für Plasmaphysik, Germany

Jolan Moldvai, Reports Library, Hungarian Academy of Sciences, Central Research Institute for Physics, Hungary

Dr. P. Kaw, Institute for Plasma Research, India

Ms. P.J. Pathak, Librarian, Institute for Plasma Research, India

Ms. Clelia De Palo, Associazione EURATOM-ENEA, Italy

Dr. G. Grosso, Instituto di Fisica del Plasma, Italy

Librarian, Naka Fusion Research Establishment, JAERI, Japan

Library, Laboratory for Complex Energy Processes, Institute for Advanced Study, Kyoto University, Japan

Research Information Center, National Institute for Fusion Science, Japan

Dr. O. Mitarai, Kyushu Tokai University, Japan

Dr. Jiangang Li, Institute of Plasma Physics, Chinese Academy of Sciences, People's Republic of China

Professor Yuping Huo, School of Physical Science and Technology, People's Republic of China

Library, Academia Sinica, Institute of Plasma Physics, People's Republic of China

Librarian, Institute of Physics, Chinese Academy of Sciences, People's Republic of China

Dr. S. Mirnov, TRINITI, Troitsk, Russian Federation, Russia

Dr. V.S. Strelkov, Kurchatov Institute, Russian Federation, Russia

Professor Peter Lukac, Katedra Fyziky Plazmy MFF UK, Mlynska dolina F-2, Komenskeho Univerzita, SK-842 15 Bratislava, Slovakia

Dr. G.S. Lee, Korea Basic Science Institute, South Korea

Institute for Plasma Research, University of Maryland, USA

Librarian, Fusion Energy Division, Oak Ridge National Laboratory, USA

Librarian, Institute of Fusion Studies, University of Texas, USA

Librarian, Magnetic Fusion Program, Lawrence Livermore National Laboratory, USA

Library, General Atomics, USA

Plasma Physics Group, Fusion Energy Research Program, University of California at San Diego, USA

Plasma Physics Library, Columbia University, USA

Alkesh Punjabi, Center for Fusion Research and Training, Hampton University, USA

Dr. W.M. Stacey, Fusion Research Center, Georgia Institute of Technology, USA

Dr. John Willis, U.S. Department of Energy, Office of Fusion Energy Sciences, USA

Mr. Paul H. Wright, Indianapolis, Indiana, USA 
The Princeton Plasma Physics Laboratory is operated by Princeton University under contract with the U.S. Department of Energy.

\author{
Information Services \\ Princeton Plasma Physics Laboratory \\ P.O. Box 451 \\ Princeton, NJ 08543
}

Phone: 609-243-2750

Fax: 609-243-2751

e-mail: pppl_info@pppl.gov

Internet Address: http://www.pppl.gov 